\title{
Response to inhaled loxapine in patients with schizophrenia or bipolar I disorder: PANSS-EC responder analyses
}

\author{
Scott Zeller, Leslie Zun, James V. Cassella, Daniel A. Spyker and Paul P. Yeung
}

\section{Background}

Efficacy of inhaled loxapine 5 or $10 \mathrm{mg}$ in treating agitation was shown using the Positive and Negative Syndrome Scale Excited Component (PANSS-EC) in two Phase III randomised, double-blind, placebo-controlled trials in 344 agitated patients with schizophrenia and 314 patients with bipolar I disorder (Clinicaltrials.gov: NCT00628589, NCT00721955).

\section{Aims}

To examine the five individual items comprising the PANSS-EC and the percentage of patients achieving a clinical response (reduction of $\geq 40 \%$ ) in PANSS-EC (Response-40) for these two studies.

\section{Method}

Response- 40 was examined at the primary end-point $(2 \mathrm{~h})$ and over time.

\section{Results}

Response-40 and each PANSS-EC item score were statistically significant $\mathrm{v}$. placebo at $2 \mathrm{~h}$ and at each assessment time point for both doses.

\section{Conclusions}

Inhaled loxapine produced rapid improvement in agitated patients with schizophrenia or bipolar I disorder, achieving Response-40 at the first assessment (10 min post dose). These results highlight the effectiveness of loxapine across all components of agitation as measured by the PANSS-EC.

\section{Declaration of interest}

S.Z. is a member of the speakers bureau for Grupo Ferrer. L.Z. has been a speaker and grant recipient for Teva Pharmaceuticals. J.V.C. and D.A.S. were employees of Alexza Pharmaceuticals during execution of the studies, and are currently paid consultants for and have received stock and/ or stock options from Alexza Pharmaceuticals. P.P.Y. is a full-time employee and receives stock options from Teva Pharmaceuticals.

\section{Copyright and usage}

(C) The Royal College of Psychiatrists 2017. This is an open access article distributed under the terms of the Creative Commons Non-Commercial, No Derivatives (CC BY-NC-ND) license.
Psychomotor agitation, defined as excessive motor and verbal activity associated with a feeling of inner tension, according to the American Psychiatric Association's DSM-5, ${ }^{1}$ is commonly associated with a number of different psychiatric disorders, including schizophrenia and bipolar I disorder. ${ }^{2,3}$ Agitation may escalate at times, even to the point of necessitating physical restraint or seclusion. ${ }^{4}$ In busy emergency departments, the speed of treatment onset is generally considered the most important criterion when selecting anti-agitation medication. ${ }^{2}$

There are currently several treatments and formulations available for treating agitation in patients with psychiatric illnesses. ${ }^{5} \mathrm{Al}-$ though oral loxapine is an established treatment for schizophrenia, the intramuscular formulation has been used in some countries to treat agitation. ${ }^{6}$ The intramuscular loxapine formulation was previously approved and marketed but is no longer available in the USA. However, an inhaled formulation of loxapine (Adasuve ${ }^{\circledR}$, Alexza Pharmaceuticals, Mountain View, California, USA) approved by the US Food and Drug Administration is available for the treatment of agitation in patients with schizophrenia or bipolar I disorder. ${ }^{7,8}$

The effects of the inhaled formulation of loxapine on agitation in patients with schizophrenia and bipolar I disorder have been investigated in two Phase III clinical trials (Clinicaltrials.gov: NCT00628589, NCT00721955). ${ }^{9,10}$ Notable results from the two studies include statistically significant reductions in the primary outcome measure, the Positive and Negative Syndrome Scale Excited Component (PANSS-EC) score, $v$. placebo at $2 \mathrm{~h}$ post administration. These statistically significant reductions $(P<0.0001)$ were observed in PANSS-EC scores 10 min post administration, the earliest assessment time point in both studies. This is substantially earlier than the observed onset of the pharmacological effects for the oral or intramuscular loxapine formulations $(<30 \mathrm{~min}){ }^{11}$ Furthermore, statistically significant changes in the Clinical Global Impression - Improvement (CGI-I) score $(P<0.0001)$ demonstrated clinically relevant improvements, with more patients in the loxapine-treated groups classified as much improved and very much improved compared with the placebo group. ${ }^{9,10}$ Citrome examined the effect size in these two studies over time. ${ }^{11}$

PANSS-EC (also known as PEC) represents a simple clinical scale used to assess agitation level in patients. ${ }^{12}$ PANSS-EC is part of the PANSS scale, a more comprehensive measure that includes an additional four components: negative, positive, disorganised (cognitive) and depression anxiety. ${ }^{13}$ The PANSS-EC scale is used in clinical trials and comprises five items associated with agitation: poor impulse control, tension, hostility, uncooperativeness and excitement. The PANSS-EC score is the sum of these five items. The PANSS-EC score has not been used regularly in clinical settings, but it has become the accepted assessment for agitation in recent pivotal clinical trials for recently approved agitation treatments. ${ }^{14}$ Recently, the validity and ability of the PANSS-EC scale to detect changes in agitated patients has been demonstrated, together with a strong linear correlation with scales such as CGI Severity. ${ }^{12}$

Analyses of the different assessment scales to validate their usage in assessing agitation has indicated that a $38 \%$ reduction in PANSS-EC score correlates to a CGI-I rating of much improved. ${ }^{12}$ Thus, a $40 \%$ reduction in PANSS-EC score has been considered a clinically relevant reduction in similar studies of antipsychotics. ${ }^{15,16}$ 
Defining a responder as a patient with achievement of a specified (clinically meaningful) reduction in PANSS-EC score, and examining the percentage of patients achieving this reduction, can provide a clinical standard for PANSS-EC reduction in the treatment of agitation and facilitate the comparison of efficacy between different antipsychotics.

We performed a post hoc analysis of the results from the two Phase III clinical studies ${ }^{9,10}$ to determine the percentage of patients achieving a clinical response, defined as a reduction of $\geq 40 \%$ in PANSS-EC score. We also examined the individual items of the PANSS-EC (poor impulse control, tension, hostility, uncooperativeness and excitement) to assess how each responded to treatment and contributed to the total (PANSS-EC) over time.

\section{Method}

\section{Study design}

The analyses presented here comprise data from two previously described Phase III trials of inhaled loxapine (Clinicaltrials.gov: NCT00628589, NCT00721955). ${ }^{9,10}$ These studies demonstrated the safety, efficacy and tolerability of 5 and $10 \mathrm{mg}$ doses of inhaled loxapine for the treatment of acute agitation in patients with schizophrenia ${ }^{9}$ or bipolar I disorder..$^{10}$ The doses used in these studies were selected based on previous clinical research showing that doses $\leq 10 \mathrm{mg}$ were well tolerated and demonstrated superiority to placebo in reducing agitation. ${ }^{17,18}$

The Phase III clinical trials of inhaled loxapine were multicentre, randomised, double-blind, placebo-controlled, parallelgroup and repeat-dose (up to three doses if required) trials conducted in the USA. The studies were designed and performed in accordance with the International Conference on Harmonisation E6 Good Clinical Practice Guidelines, the Declaration of Helsinki and US Food and Drug Administration and European Union guidelines. Independent institutional review boards approved the studies, and all patients provided written informed consent.

\section{Description of patients}

Eligible patients were males and females aged 18-65 years, otherwise in generally good health, diagnosed with either schizophrenia or bipolar I disorder based on DSM-IV-TR criteria ${ }^{19}$ by a research-trained psychiatrist. The patients were clinically agitated at baseline according to PANSS-EC score. Eligible patients had a PANSS-EC score $\geq 14$, with at least one of the five items rated $\geq 4$.

The PANSS-EC is scored by summing the ratings of the five items associated with agitation (poor impulse control, tension, hostility, uncooperativeness and excitement), rated on a scale from 1 (absent) to 7 (extreme). Total scores thus range from 5 to $35,20,21$ and scores $\geq 20$ correspond to clinically severe agitation. ${ }^{12}$

\section{Randomisation, treatment and assessments}

Patients were randomised 1:1:1 to inhaled loxapine $5 \mathrm{mg}$, inhaled loxapine $10 \mathrm{mg}$ or placebo. Assessments were performed at baseline and at 10, 20,30, 45, 60 and $90 \mathrm{~min}$ and 2,4 and $24 \mathrm{~h}$ following the initial dose. If required, a second dose was allowed following completion of the 2-h assessment, and a third dose was allowed at or after $4 \mathrm{~h}$ following dose 2 . Lorazepam rescue was permitted at any time after dose 2 .

\section{Drug administration}

Inhaled loxapine was delivered via the Staccato ${ }^{\circledast}$ system (Alexza Pharmaceuticals, Mountain View, California, USA), described in detail elsewhere. ${ }^{9,22}$ Briefly, it is a hand-held drug device that facilitates rapid systemic delivery of loxapine via inhalation of a thermally generated aerosol with intravenous-like kinetics and a median time to maximum concentration of $2 \mathrm{~min} .{ }^{22}$ Oral inhalation through the device triggers the controlled rapid heating of a thin film of excipient-free loxapine to form a pure-drug vapour.

\section{Clinical end-points}

The original primary end-point of the studies included in this analysis was change from baseline in PANSS-EC score $2 \mathrm{~h}$ post dose (5 or $10 \mathrm{mg}$ ) compared with placebo. Secondary end-points were change from baseline in PANSS-EC score at each assessment time point up to $2 \mathrm{~h}$, change from baseline in PANSS-EC score stratified by median baseline PANSS-EC score and increase in the CGI-I responder analysis.

The current post hoc analysis of the PANSS-EC scores evaluated the change from baseline for each individual PANSS-EC subscale item (poor impulse control, tension, hostility, uncooperativeness and excitement) and the percentage of PANSS-EC responders (patients achieving $\geq 40 \%$ improvement over baseline scores, defined as Response-40) at each assessment time point.

\section{Statistical analysis}

The efficacy population included all patients who received any study drug and had both a baseline assessment and at least one post-dose efficacy assessment. Statistical testing of the post hoc endpoints used a two-way non-parametric analysis of covariance by ranks (within strata). PANSS-EC responder analysis comparisons between the treatment and placebo groups were calculated by the Cochran-Mantel-Haenszel test (pairwise) using SAS software version 9.2 (SAS Institute, Cary, North Carolina, USA). Forest plots and confidence intervals were calculated using StatsDirect version 2.8.0 (StatsDirect, Cheshire, UK).

The number needed to treat (NNT) to achieve a response was calculated to help place the response results into clinical context. NNT was calculated as $1 /(\%$ response rate for treated $-\%$ response rate for control).

\section{Results}

\section{Patient disposition}

In the schizophrenia study, 344 patients received at least one dose of study drug, of whom 338 completed the study. ${ }^{9}$ In the bipolar I disorder study, 314 patients received at least one dose of study drug and 312 patients completed the study. ${ }^{10}$ Patient baseline characteristics for both studies are shown in Table 1 .

\section{PANSS-EC responders}

A reduction in agitation of $\geq 40 \%$ in PANSS-EC score (Response- 40 ) was observed in $\sim 20 \%$ of patients with schizophrenia and those with bipolar I disorder at 10 min post loxapine dose (Fig. 1). This reduction was observed in patients receiving both the 5 and $10 \mathrm{mg}$ doses. The percentage of patients achieving Response- 40 increased with time, reaching a peak of $\sim 70 \%$ in the $10-\mathrm{mg}$ dose group for both the schizophrenia and bipolar I disorder groups.

The percentages of PANSS-EC responders in the two loxapine dose groups (5 and $10 \mathrm{mg}$ ) were statistically significantly higher 


\begin{tabular}{|c|c|c|c|c|c|c|}
\hline \multirow[b]{2}{*}{ Characteristic } & \multicolumn{3}{|c|}{ Patients with schizophrenia } & \multicolumn{3}{|c|}{ Patients with bipolar I disorder } \\
\hline & $\begin{array}{c}\text { Placebo } \\
n=115\end{array}$ & $\begin{array}{c}5 \mathrm{mg} \\
n=116\end{array}$ & $\begin{array}{l}10 \mathrm{mg} \\
n=113\end{array}$ & $\begin{array}{c}\text { Placebo } \\
n=105\end{array}$ & $\begin{array}{c}5 \mathrm{mg} \\
n=104\end{array}$ & $\begin{array}{l}10 \mathrm{mg} \\
n=105\end{array}$ \\
\hline Mean (s.d.) age, years & $48.3(9.5)$ & $43.2(10.2)$ & $42.1(9.8)$ & $40.6(9.8)$ & $41.2(9.6)$ & $40.5(9.8)$ \\
\hline Mean (s.d.) time since diagnosis, years & $18.8(10.3)$ & $16.5(10.8)$ & $18.2(10.0)$ & $12.0(10.1)$ & $12.8(8.9)$ & $11.7(9.1)$ \\
\hline Mean (s.d.) duration of current agitation episode, days & $6.9(9.2)$ & $6.1(7.5)$ & $7.6(11.5)$ & $14.2(21.5)$ & $16.0(32.4)$ & $9.7(10.2)$ \\
\hline Mean (s.d.) number of previous hospitalisations & $9.6(9.0)$ & $9.2(12.2)$ & $9.7(11.3)$ & $5.9(6.6)$ & $5.5(6.6)$ & $5.0(6.4)$ \\
\hline Male, \% & 70 & 77 & 75 & 53 & 45 & 51 \\
\hline Smokers, \% & 78 & 81 & 86 & 74 & 76 & 73 \\
\hline \multicolumn{7}{|l|}{ Mean (s.d.) baseline score on items of the PANSS-EC scale } \\
\hline Poor impulse control & $3.3(0.6)$ & $3.4(0.8)$ & $3.4(0.6)$ & $3.3(0.9)$ & $3.4(0.8)$ & $3.3(0.8)$ \\
\hline Tension & $4.0(0.6)$ & $4.0(0.6)$ & $4.0(0.7)$ & $4.0(0.9)$ & $4.1(0.7)$ & $4.2(0.6)$ \\
\hline Hostility & $3.2(0.8)$ & $3.3(0.8)$ & $3.3(0.8)$ & $3.5(1.0)$ & $3.3(1.0)$ & $3.2(0.9)$ \\
\hline Uncooperativeness & $3.0(0.8)$ & $3.2(0.8)$ & $3.2(0.9)$ & $2.9(0.9)$ & $2.8(0.8)$ & $2.7(1.0)$ \\
\hline Excitement & $3.7(0.7)$ & $3.9(0.8)$ & $3.7(0.7)$ & $4.0(0.9)$ & $3.9(0.7)$ & $3.9(0.7)$ \\
\hline
\end{tabular}
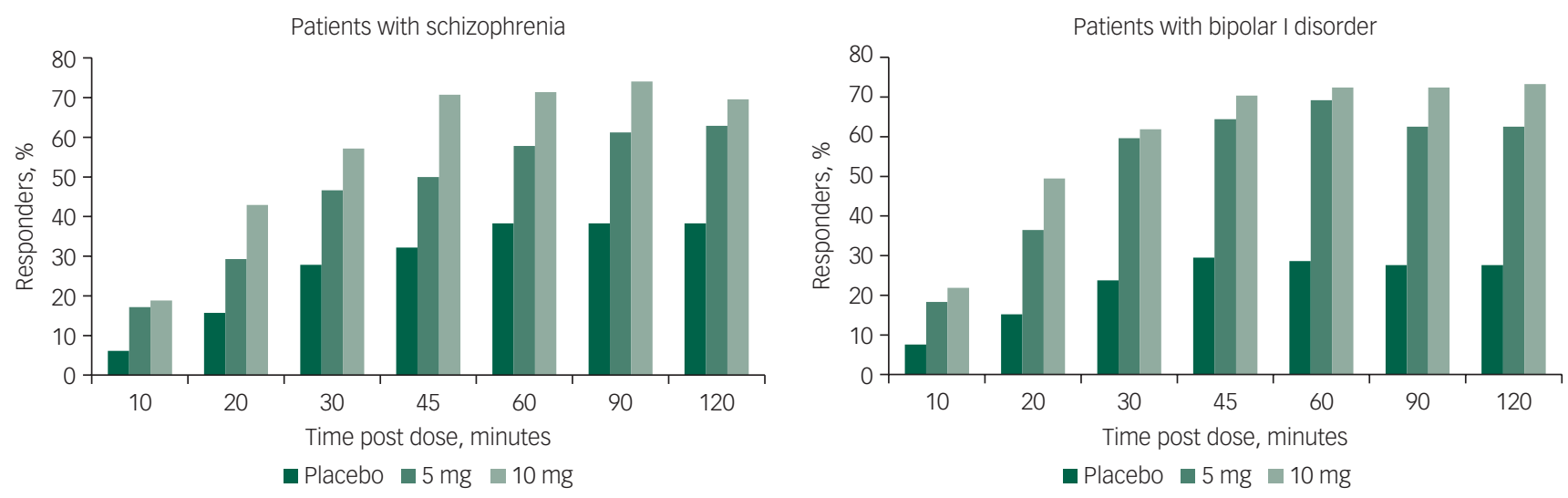

Fig. 1 PANSS-EC response over time: patients with $\geq 40 \%$ PANSS-EC score reduction. PANSS-EC, Positive and Negative Syndrome Scale - Excited Component.

than placebo at the $10 \mathrm{~min}$ time point $(P=0.0056$ and $P=0.0012$ for the schizophrenia study; $P=0.0059$ and $P=0.0017$ for the bipolar I disorder study). Statistical significance $v$. placebo was maintained through all time points through $2 \mathrm{~h}$ for both the 5 and $10 \mathrm{mg}$ doses in both studies (Fig. 1).

Figure 2 shows the odds ratio (OR) at the $2 \mathrm{~h}$ time point for 5 and $10 \mathrm{mg}$ to placebo for each study for the CGI, PANSS-EC and each subscale. PANSS-EC OR shows a similar and slighter stronger response compared with CGI OR. The three individual items' (impulse control, tension and excitement) ORs show a similar response pattern, and all five items are statistically significant $(P<0.05)$ as indicated by the OR confidence interval (CI) exclusion of 1.0.

The NNT for the PANSS-EC at the $2 \mathrm{~h}$ time point for the 5 and $10 \mathrm{mg}$ treatment $v$. placebo was 4.05 and 3.16 for the schizophrenia study and 2.87 and 2.19 for the bipolar I disorder study.

\section{PANSS-EC items}

In both studies, there were statistically significant reductions in all five PANSS-EC items for the 5 and $10 \mathrm{mg}$ doses $v$. placebo at $2 \mathrm{~h}$ post dose (Fig. 3). Overall, score reductions in all PANSS-EC items across the two studies were between 1 and 2 units from baseline for both doses over the first $2 \mathrm{~h}$ post dose. Both the 5 and $10 \mathrm{mg}$ dose groups reduced statistically significantly $(P<0.05)$ for all PANSS-EC item scores $v$. placebo at each time point through $2 \mathrm{~h}$ (Fig. 3), with the exception of the uncooperativeness subscale, where $P=0.0853$ for the $5 \mathrm{mg}$ dose at $10 \mathrm{~min}$ in the schizophrenia study.

\section{Discussion}

The present post hoc analysis demonstrates that a statistically significantly greater percentage of patients achieved $\geq 40 \%$ reduction in agitation assessed by PANSS-EC score at $2 \mathrm{~h}$ following administration of inhaled loxapine at both the 5 and $10 \mathrm{mg}$ doses, compared with those who received placebo. This result was observed in both the schizophrenia and bipolar I disorder populations studied.

The percentage of patients who achieved $\geq 40 \%$ reduction in PANSS-EC score in the loxapine-treated groups was statistically significantly greater $v$. placebo as early as the 10-minute post-dose assessment, confirming the rapid onset of effect seen in the original clinical trials. ${ }^{9,10}$ 


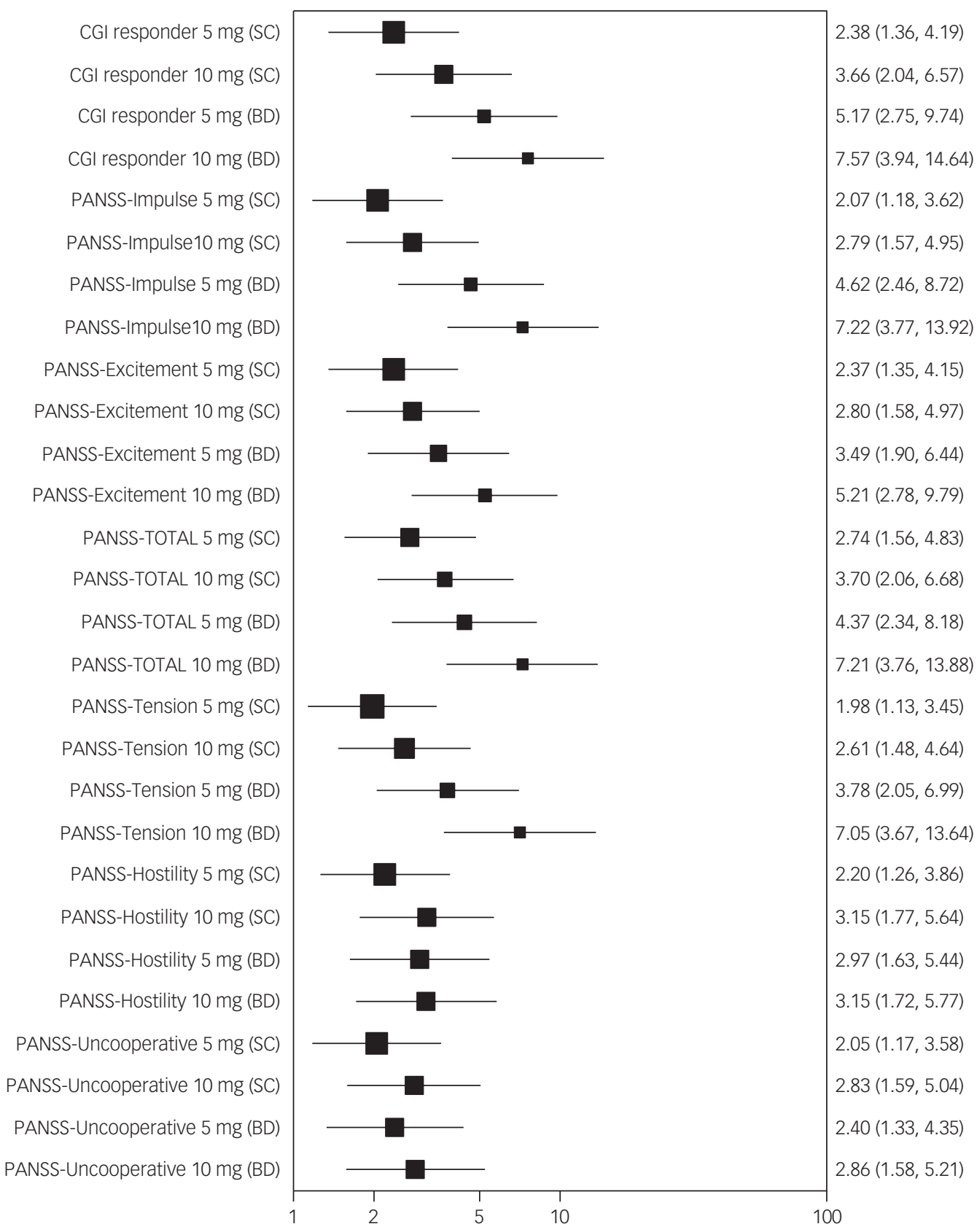

Fig. 2 Odds ratio (OR) forest plot for responders at $2 \mathrm{~h}$ : CGI responders, total PANSS-EC scores and individual PANSS-EC subscale scores. BD, bipolar I disorder; Cl, confidence interval; CGI, Clinical Global Impression scale; OR, odds ratio; SC, schizophrenia; PANSS-EC, Positive and Negative Syndrome Scale - Excited Component. All ORs are statistically significant (95\% Cl excludes 1.0).

Analysis of the individual items of the PANSS-EC scale show statistically significant reductions in scores for each of the five items $v$. placebo as early as $10 \mathrm{~min}$ post dose and at the $2 \mathrm{~h}$ time point for both the 5 and $10 \mathrm{mg}$ doses of loxapine and in both studies. Thus, it can be concluded that each of the five PANSS-EC items contributed to the reduction in the PANSS-EC score observed with inhaled loxapine treatment. The total PANSS-EC score may represent a larger effect size than any individual subscale.

Comparison with similar studies on agitation management where $40 \%$ reduction in PANSS-EC score was used as an outcome $^{15,16}$ revealed that although the inclusion criteria varied among the studies, patients' baseline and demographic characteristics were similar: mean PANSS-EC scores ranged from 17.3 to 19.0 and the average score range per item was 3.5-3.8 for aripiprazole, olanzapine and inhaled loxapine. ${ }^{16}$ In a similar study of quetiapine, ${ }^{15} 50 \%$ of patients achieved $\geq 40 \%$ reduction in PANSS-EC score at $2 \mathrm{~h}$ post dose. Although a direct comparison cannot be made with the results of this analysis due to the differences in inclusion criteria and the number of patients analysed, a greater proportion of patients (70\%) achieved $\geq 40 \%$ reduction in PANSS-EC score at $2 \mathrm{~h}$ post dose with inhaled loxapine, confirming treatment efficacy.

\section{Limitations}

The post hoc analysis of the PANSS-EC results described here has several limitations. One limitation is that the treatment was performed in a controlled healthcare setting, which may not necessarily be 
Patients with schizophrenia

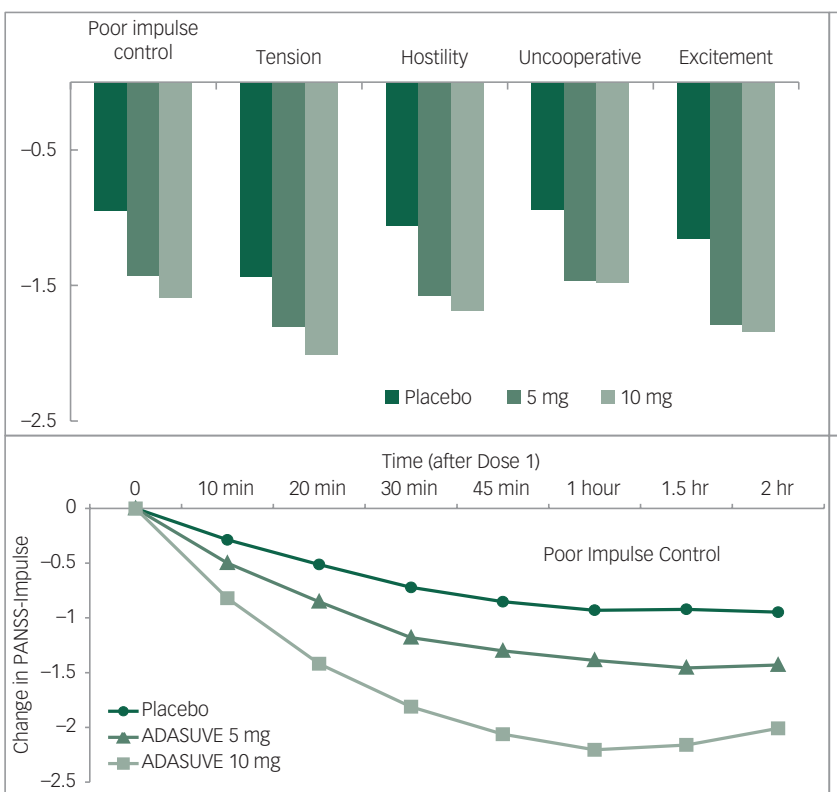

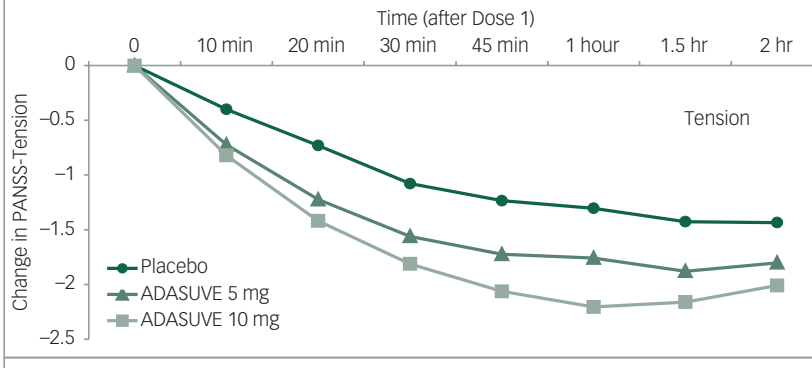
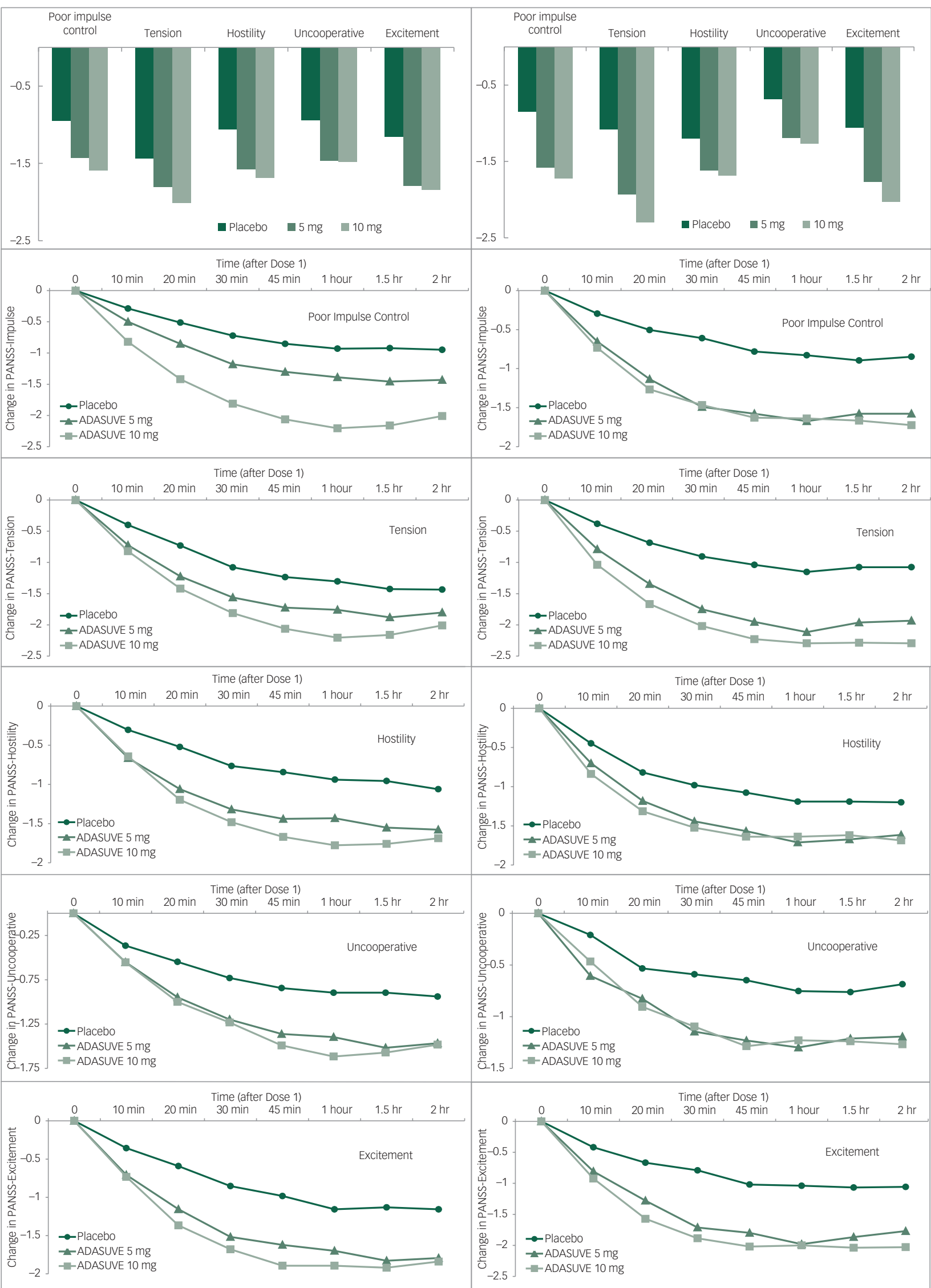

Fig. 3 Changes from baseline in individual PANSS-EC item scores at $2 \mathrm{~h}$ post dose (bar graph) and changes in individual PANSS-EC item scores over time (line graphs). PANSS-EC, Positive and Negative Syndrome Scale - Excited Component. In the individual PANSS-EC item analyses, all time points from $10 \mathrm{~min}$ to $2 \mathrm{~h}$ for both doses were statistically significant $(P<0.05)$, except for the uncooperative item $(P=0.0853$ for the $5 \mathrm{mg}$ dose at $10 \mathrm{~min}$ in the schizophrenia study). 
representative of the clinical setting where this treatment will be used. Another limitation is that patient groups provided informed consent and were screened to meet eligibility criteria; hence, patients who were too agitated to give consent were excluded. This would not be the case for the patient population who would receive treatment for agitation in a clinical setting. In addition, the application of a $40 \%$ reduction in PANSS-EC score was performed post hoc, and the clinical relevance of the $40 \%$ reduction is unclear. Nonetheless, the $\geq 40 \%$ reduction in PANSS-EC score at $2 \mathrm{~h}$ post dose was chosen to be consistent with other antipsychotic studies for similar patient groups and was based on its correlation observed with other measuring scales. ${ }^{7,8,12}$

The analysis of the Phase III clinical trials of inhaled loxapine demonstrates a rapid onset of action (within $10 \mathrm{~min}$ of administration) across all items of the PANSS-EC, confirming the results in the original Phase III studies, ${ }^{9,10}$ and highlights the effectiveness of inhaled loxapine on all components of agitation included in the PANSS-EC scale.

Scott Zeller, MD, UC Riverside School of Medicine, Riverside, California, USA; Leslie Zun, MD, Mount Sinai Hospital, Chicago, Illinois, USA; James V. Cassella, PhD, Alexza Pharmaceuticals, Mountain View, California, USA; Daniel A. Spyker, PhD, MD, Alexza Pharmaceuticals, Mountain View, California, USA; Paul P. Yeung, MD, MPH Teva Pharmaceuticals, Frazer, Pennsylvania, USA

Correspondence: Daniel A. Spyker, Alexza Pharmaceuticals, 2091 Stierlin Ct, Mountain View, CA 94043, USA. Email: dspyker@alexza.com

First received 11 Jun 2017, final revision 17 Aug 2017, accepted 24 Sep 2017

\section{Funding}

This study was funded by Alexza Pharmaceuticals. Medical writing support was funded by Teva Pharmaceuticals.

\section{Acknowledgements}

Medical writing support was provided by Paul Littlebury, PhD, of Excel Scientific Solutions, Horsham, UK.

\section{References}

1 American Psychiatric Association. Diagnostic and Statistical Manual of Mental Disorders, Fifth Edition (DSM-5). APA, 2013.

2 Alderfer BS, Allen MH. Treatment of agitation in bipolar disorder across the life cycle. J Clin Psychiatry 2003; 64 (suppl 4): 3-9.

3 Hankin CS, Bronstone A, Koran LM. Agitation in the inpatient psychiatric setting: a review of clinical presentation, burden, and treatment. J Psychiatr Pract 2011; 17: 170-85.

4 Marder SR. A review of agitation in mental illness: treatment guidelines and current therapies. J Clin Psychiatry 2006; 67 (suppl 10): 13-21.
5 Nordstrom K, Allen $\mathrm{MH}$. Alternative delivery systems for agents to treat acute agitation: progress to date. Drugs 2013; 73: 1783-92.

6 Bourdinaud V, Pochard F. Survey of management methods for patients in a state of agitation at admission and emergency departments in France [in French]. Encephale 2003; 29: 89-98.

7 Adasuve [prescribing information]. Alexza Pharmaceuticals, 2013.

8 European Medicines Agency. Adasuve (Ioxapine) Inhalation Powder: EU Summary of Product Characteristics. (http://ec.europa.eu/health/documents/ community-register/2013/20130220125343/anx_125343_en.pdf).

9 Lesem MD, Tran-Johnson TK, Riesenberg RA, Feifel D, Allen MH, Fishman R, et al. Rapid acute treatment of agitation in individuals with schizophrenia: multicentre, randomised, placebo-controlled study of inhaled loxapine. Br J Psychiatry 2011; 198: $51-8$.

10 Kwentus J, Riesenberg RA, Marandi M, Manning RA, Allen MH, Fishman RS, et al. Rapid acute treatment of agitation in patients with bipolar I disorder: a multicenter, randomized, placebo-controlled clinical trial with inhaled loxapine. Bipolar Disord 2012; 14: 31-40

11 Citrome L. Inhaled loxapine for agitation revisited: focus on effect sizes from 2 Phase III randomised controlled trials in persons with schizophrenia or bipolar disorder. Int J Clin Pract 2012; 66: 318-25.

12 Montoya A, Valladares A, Lizan L, San L, Escobar R, Paz S. Validation of the Excited Component of the Positive and Negative Syndrome Scale (PANSS-EC) in a naturalistic sample of 278 patients with acute psychosis and agitation in a psychiatric emergency room. Health Qual Life Outcomes 2011; 9: 18.

13 Emsley R, Rabinowitz J, Torreman M. The factor structure for the Positive and Negative Syndrome Scale (PANSS) in recent-onset psychosis. Schizophr Res 2003; 61: 47-57.

14 Leucht S, Kane JM, Kissling W, Hamann J, Etschel E, Engel RR. What does the PANSS mean? Schizophr Res 2005; 79: 231-8.

15 Pratts M, Citrome L, Grant W, Leso L, Opler LA. A single-dose, randomized, double-blind, placebo-controlled trial of sublingual asenapine for acute agitation. Acta Psychiatr Scand 2014; 130: 61-8.

16 Citrome L. Comparison of intramuscular ziprasidone, olanzapine, or aripiprazole for agitation: a quantitative review of efficacy and safety. J Clin Psychiatry 2007: 68: $1876-85$.

17 Cassella J, Fishman R, Spyker D. Inhaled loxapine rapidly improves acute agitation in schizophrenic patients. Presented at: American Psychiatric Association 162nd Annual Meeting, 16-21 May 2009, San Francisco, California, USA. Available at: http://www.europeanneuropsychopharmacology.com/article/ S0924-977X(10)70765-3/abstract.

18 Spyker DA, Munzar P, Cassella JV. Pharmacokinetics of loxapine following inhalation of a thermally generated aerosol in healthy volunteers. J Clin Pharmacol 2010; 50: $169-79$

19 American Psychiatric Association. Diagnostic and Statistical Manual of Mental Disorders, Fourth Edition, Text Revision (DSM-IV-TR). APA, 2000.

20 Kay SR, Fiszbein A, Opler LA. The Positive and Negative Syndrome Scale (PANSS) for schizophrenia. Schizophr Bull 1987; 13: 261-76.

21 Kay SR. Significance of the positive-negative distinction in schizophrenia. Schizophr Bull 1990; 16: 635-52.

22 Dinh K, Myers DJ, Noymer PD, Cassella JV. In vitro aerosol characterisation of Staccato@ Loxapine. Int J Pharm 2011; 403: 101-8. 